\title{
Feasibility of frameless single-fraction stereotactic radiosurgery for spinal lesions
}

\author{
Peter C. Gerszten, M.D., M.P.H., Cihat Ozhasoglu, Ph.D., Steven A. Burton, M.D., \\ Shalom Kalnicki, M.D., and William C. Welch, M.D. \\ Departments of Neurological Surgery and Radiation Oncology, University of Pittsburgh School of \\ Medicine, UPMC Health System, Pittsburgh, Pennsylvania
}

\begin{abstract}
Object. The role of stereotactic radiosurgery for the treatment of intracranial lesions is well established. Its use for the treatment of spinal lesions has been limited by the availability of effective target-immobilizing devices. In this study the authors evaluated the CyberKnife Real-Time Image-Guided Radiosurgery System for spinal lesion treatment involving a single-fraction radiosurgical technique.

Methods. This frameless image-guided radiosurgery system uses the coupling of an orthogonal pair of x-ray cameras to a dynamically manipulated robot-mounted linear accelerator possessing six degrees of freedom, which guides the therapy beam to the target without the use of frame-based fixation. Cervical lesions were located and tracked relative to osseous skull landmarks; lower spinal lesions were tracked relative to percutaneously placed gold fiducial bone markers. Fifty-six spinal lesions in 46 consecutive patients were treated using single-fraction radiosurgery ( 26 cervical, 15 thoracic, and 11 lumbar, and four sacral). There were 11 benign and 45 metastatic lesions.

Tumor volume ranged from 0.3 to $168 \mathrm{ml}$ (mean $26.7 \mathrm{ml}$ ). Thirty-one lesions had previously received external-beam radiotherapy with maximum spinal cord doses. Dose plans were calculated based on computerized tomography scans acquired using $1.25-\mathrm{mm}$ slices. Tumor dose was maintained at 12 to $18 \mathrm{~Gy}$ to the $80 \%$ isodose line; spinal cord lesions receiving greater than 8 Gy ranged from 0 to $1.3 \mathrm{ml}$ (mean $0.3 \mathrm{ml}$ ). All patients tolerated the procedure in an outpatient setting. No acute radiation-induced toxicity or new neurological deficits occurred during the follow-up period. Axial and radicular pain improved in all patients who were symptomatic prior to treatment.

Conclusions. Spinal stereotactic radiosurgery involving a frameless image-guided system was found to be feasible and safe. The major potential benefits of radiosurgical ablation of spinal lesions are short treatment time in an outpatient setting with rapid recovery and symptomatic response. This procedure offers a successful alternative therapeutic modality for the treatment of a variety of spinal lesions not amenable to open surgical techniques; the intervention can be performed in medically untreatable patients, lesions located in previously irradiated sites, or as an adjunct to surgery.
\end{abstract}

\section{KEY WORDS • CyberKnife • image-guided surgery • metastasis • radiosurgery • robotic surgery $\bullet$ spinal tumor $\bullet$ stereotaxis}

During the past decade, stereotactic radiosurgery has emerged as a well-established treatment for a number of intracranial neurosurgical disease processes. The role of stereotactic radiosurgery for the treatment of a wide variety of benign and malignant intracranial lesions is also well established. ${ }^{7,8}$ Stereotactic radiosurgery is an effective treatment for brain metastases, either with or without whole-brain radiotherapy, with an 85 to $95 \%$ control rate. ${ }^{8,13,24,26}$ Its role in the primary treatment of benign lesions, including arteriovenous malformations, pituitary adenomas, acoustic neuromas, and meningiomas is well supported by the literature. . $, 11,14,22,23,34^{2}$

The role of radiotherapy in the treatment of spinal tumors has been well documented. Conventional externalbeam radiotherapy lacks the precision to allow delivery of

Abbreviations used in this paper: $\mathrm{CT}=$ computerized tomography; LINAC = linear accelerator. large doses of radiation near radiosensitive structures such as the spinal cord. The low tolerance of the spinal cord to radiation often limits the treatment dose to a level far below the optimal therapeutic dose..$^{12,25,33}$ If the radiation dose could be confined more precisely to the treatment volume, as is the case in intracranial radiosurgery, the likelihood of successful tumor control would likely increase at the same time that the risk of spinal cord injury is minimized.

Current frame-based stereotactic radiosurgery devices, equipped with skull fixation devices, do not have the capability to treat lesions below the foramen magnum. Conformal radiotherapy and intensity-modulated radiotherapy are limited by problems with target immobilization; this precludes large single-fraction treatment of spinal lesions. Conventional frame-based devices used for stereotactic radiosurgery in cases of intracranial lesions rely on a rigid frame to immobilize the lesion at a known location in space. The frame acts as a fiducial reference 
system to provide accurate targeting and delivery of the radiation dose. Intracranial radiosurgery is practical because the lesions are fixed with respect to the cranium, which can be immobilized rigidly in a stereotactic frame. Although spinal lesions also have a fixed relationship to the spine, LINAC-based stereotactic radiosurgery techniques developed for spinal lesions require the direct placement of an invasive rigid external frame system onto the spine. ${ }^{17}$

A new image-guided frameless stereotactic radiosurgery delivery system known as the CyberKnife (Accuray, Inc., Sunnyvale, CA) has been developed that was approved by the United States Food and Drug Administration in 2001 for use throughout the entire spine. The system consists of a lightweight LINAC mounted on a robotic arm. Real-time image tracking allows for the tracking of patient movement with a 1-mm spatial accuracy. ${ }^{4,733}$ The CyberKnife was developed as a noninvasive means to align treatment beams precisely with targets. The system differs from conventional frame-based radiosurgery in three fundamental ways. ${ }^{33}$ First, it references the position of the treatment target to internal radiographically determined features such as the skull or implanted fiducials rather than a frame. Second, it uses real-time radiographic imaging to establish the position of the lesion during treatment and then dynamically brings the radiation beam into alignment with the observed position of the treatment target. Third, it aims each beam independently, without a fixed isocenter. Intraprocedural changes in patient positioning are compensated for by adaptive beam pointing rather than controlled through rigid immobilization. This allows the patient to be positioned in the treatment room without precise reproduction of the position in the treatment planning study.

The CyberKnife was first developed for treatment of brain tumors at Stanford University. Since 1994, the device has been used at a number of sites around the world to treat a variety of benign and malignant intracranial lesions. ${ }^{3,4,9}$ As expected, treatment outcomes have closely mirrored those obtained using conventional frame-based radiosurgery. ${ }^{33}$ With the ability to treat lesions outside of the skull because of fiducial tracking, a growing interest in using the CyberKnife to treat spinal lesions has emerged. ${ }^{30,33}$

The purpose of this study was to evaluate feasibility, clinical response, and safety by using the CyberKnife radiosurgical technique to treat spinal lesions. Because of the spatial precision with which the CyberKnife can administer radiation, it is theoretically feasible to administer a tumoricidal radiation dose in a single outpatient treatment. By minimizing the radiation delivered to surrounding healthy tissue, it should also be possible to decrease the rate of complications.

\section{CLINICAL MATERIAL AND METHODS}

\section{Patient Population}

This study involved the prospective evaluation of 46 patients in whom spinal lesions were treated using the $\mathrm{Cy}-$ berKnife system and Dynamic Tracking System (version 3.0) software. All patients were treated at the University of Pittsburgh Medical Center, and the protocol was ap- proved by the institutional review board. There were 21 men and 25 women ( $>18$ years of age). Tables 1 and 2 provide summaries of patient characteristics and lesions. The lesions included a variety of both primary and metastatic pathological entities.

\section{CyberKnife System}

The CyberKnife consists of a computer-controlled, compact source of high energy $\mathrm{x}$-ray beams; it is smaller and lighter in weight than LINACs used in conventional radiotherapy. ${ }^{2,16,31}$ Its smaller size allows it to be mounted on a computer-controlled six-axis robotic manipulator that permits a much wider range of beam orientations than can be obtained using conventional radiotherapy devices. In the CyberKnife system image-guided frameless robotic radiosurgery is involved. Two diagnostic radiography cameras are positioned orthogonally to acquire intraoperative real-time images of internal anatomy. The images are processed automatically to identify radiographic features and then registered to the treatment planning study to measure the position of the treatment site. The measured position is communicated through a real-time control loop to a robotic manipulator that points a compact $6-\mathrm{mV}$ LINAC. The system can adapt intraoperatively to changes in patient position by acquiring targeting images repeatedly and then adjusting the direction of the treatment beam. The target to be treated is identified on planning images prior to treatment, and between 60 and 100 beams are used to irradiate the target site in a stereotactic fashion. The treatment beams can be maneuvered and pointed nearly anywhere in space. Because treatment beams are also not confined to isocentric geometry, they can be arranged in complex overlapping patterns that conform to irregularly shaped tumor volumes. ${ }^{33}$

\section{Inclusion and Exclusion Criteria}

All patients were 18 years of age or older. All patients harbored histologically confirmed carcinoma from the primary site if felt to be a metastatic lesion. Table 3 provides

TABLE 1

Summary of characteristics in 46 patients

\begin{tabular}{lc}
\hline \multicolumn{1}{c}{ Characteristic } & No. of Cases \\
\hline multiple treated lesions & 10 \\
previous external-beam irradiation & 31 \\
primary indications for radiosurgery & \\
pain & 31 \\
progressive neurological deficit & 9 \\
primary modality & 3 \\
radiation boost & 3 \\
levels treated & \\
$\quad$ cervical & 26 \\
thoracic & 15 \\
lumbar & 11 \\
$\quad$ sacral & 4 \\
skull tracking & 26 \\
fiducial tracking & 30 \\
additional characteristics & \\
mean tumor volume (ml) & 26.7 \\
mean dose to $80 \%$ isodose line $(\mathrm{Gy})$ & 14 \\
mean volume of spinal cord/canal dose $>8 \mathrm{~Gy}(\mathrm{ml})$ & 0.3 \\
\hline
\end{tabular}


TABLE 2

Characteristics of 56 lesions in the 46 patients

\begin{tabular}{cc}
\hline \hline \multicolumn{1}{c}{ Tumor Type } & No. of Lesions \\
\hline benign & \\
neurofibroma & 4 \\
schwannoma & 2 \\
meningioma & 2 \\
hemangioma & 1 \\
chordoma & 1 \\
paraganglioma & 1 \\
metastatic & 14 \\
breast & 9 \\
renal & 6 \\
lung & 4 \\
hemangioblastoma & 3 \\
colon & 2 \\
prostate & 2 \\
squamous cell (laryngeal) & 1 \\
pancreas & 1 \\
osteosarcoma & 1 \\
carcinoid & 1 \\
multiple myeloma & 1 \\
clear cell & \\
\hline
\end{tabular}

a summary of the indications for spinal stereotactic radiosurgery. Exclusion criteria included: 1) evidence of overt spinal instability; 2) neurological deficit resulting from osseous compression of neural structures; or 3) previous irradiation at the tolerance dose of the spinal cord.

\section{Overview of Treatment}

The CyberKnife spinal radiosurgery treatment consists of three distinct components: 1) acquisition of CT scans based on skull osseous landmarks or implanted bone fiducials; 2) treatment planning; and 3) the treatment itself. Intracranial and cervical lesions were tracked relative to osseous skull landmarks. All other lesions were tracked relative to bone fiducials placed adjacent to the lesion. Because these implanted fiducials have a fixed relationship with the bone in which they are implanted, any movement in the vertebrae would be detected as movement in the fiducials, and this movement is detected and compensated for by the CyberKnife system.

All patients with cervical lesions were fitted with a noninvasive molded face mask (WRF/Aquaplast Corp., Wyckoff, NJ) that stabilized the head and neck on a radiographically transparent headrest. Computerized tomography scans were acquired using $1.25-\mathrm{mm}$-thick slices from the top of the skull to the bottom of the cervical spine. In cases of all other lesions, fluoroscopy was used to guide the percutaneous placement of four to six gold fiducial markers (Alpha-Omega Services, Inc., Bellflower, CA) into the pedicles immediately adjacent to the lesion; a standard Jamshidi bone marrow biopsy needle (Allegiance Healthcare Corporation, McGraw Park, IL) was used (Fig. 1 upper left and right). Three fiducials are required to define a full spatial transformation in all $6^{\circ}$ of target translation and rotation. The placement of fiducials was performed in the operating room in an outpatient setting. There were no associated complications. As an outpatient, the patient also returned for the planning CT studies. The patient was placed supine in a conformal alpha cradle during CT scanning as well as during treatment. Scans were acquired using $1.25-\mathrm{mm}$-thick slices to include the lesion of interest as well as all fiducials.

The second component of the radiosurgical treatment is the development of the treatment plan. Each treatment plan was devised jointly by a team of neurosurgeons, radiation oncologists, and radiation physicists. In each case, the radiosurgical treatment plan was designed based on tumor geometry, proximity to spinal cord, and location. Treatment planning was performed using the Accuray treatment planning system DTS 3.0 (Fig. 1 lower left and right). The tumor dose was determined based on the histological character of the tumor, spinal cord tolerance, and previous levels radiation doses.

The third component of the CyberKnife treatment is the actual treatment delivery. All procedures were performed using a single-fraction in an outpatient setting. The patients were placed supine on the CyberKnife treatment table with the appropriate immobilization device. Several of the patients with thoracic or lumbar lesions localized with fiducials were actually more comfortable without the alpha cradle, and only pillows were used. During the treatment, real-time digital radiographic images were obtained. The location of the vertebral body being treated was established on these images and used to determine tumor location as previously described. The patient was observed throughout the treatment by closed-circuit television.

\section{RESULTS}

\section{Treatment Planning}

Table 1 provides a summary of patient characteristics. The tumor dose was maintained at 1200 to $1800 \mathrm{cGy}$ to the $80 \%$ isodose line contoured at the edge of the target volume. The maximum intratumoral dose ranged from 1200 to 2000 cGy (mean 1688 cGy). In all patients, the entire spinal canal was treated as the spinal cord, even in cases of lumbar and sacral lesions. A limit of $800 \mathrm{cGy}$ was set as the maximum spinal cord dose for treatment planning calculations. The spinal cord volume receiving greater than $800 \mathrm{cGy}$ ranged from 0 to $1.3 \mathrm{ml}$ (mean $0.3 \mathrm{ml}$ ). A limit of $200 \mathrm{cGy}$ was set as the maximum dose to each of the kidneys. Twenty-six cases involved the use of skull osseous landmarks for image guidance, and 30 cases involved the use of fiducial tracking.

The dose to the tumor margin was based on tumor type, location, and history of prior fractionated radiotherapy. Preoperatively records regarding previous spinal cord irradiation were carefully considered. Thirty-one lesions

TABLE 3

Candidate lesions for CyberKnife stereotactic radiosurgery

well-circumscribed lesions

minimal spinal cord compromise

previously irradiated lesions

recurrent surgical lesions

lesions requiring difficult surgical approaches

relatively short life expectancy as exclusion criterion for open surgery

significant medical comorbidities precluding open surgery 


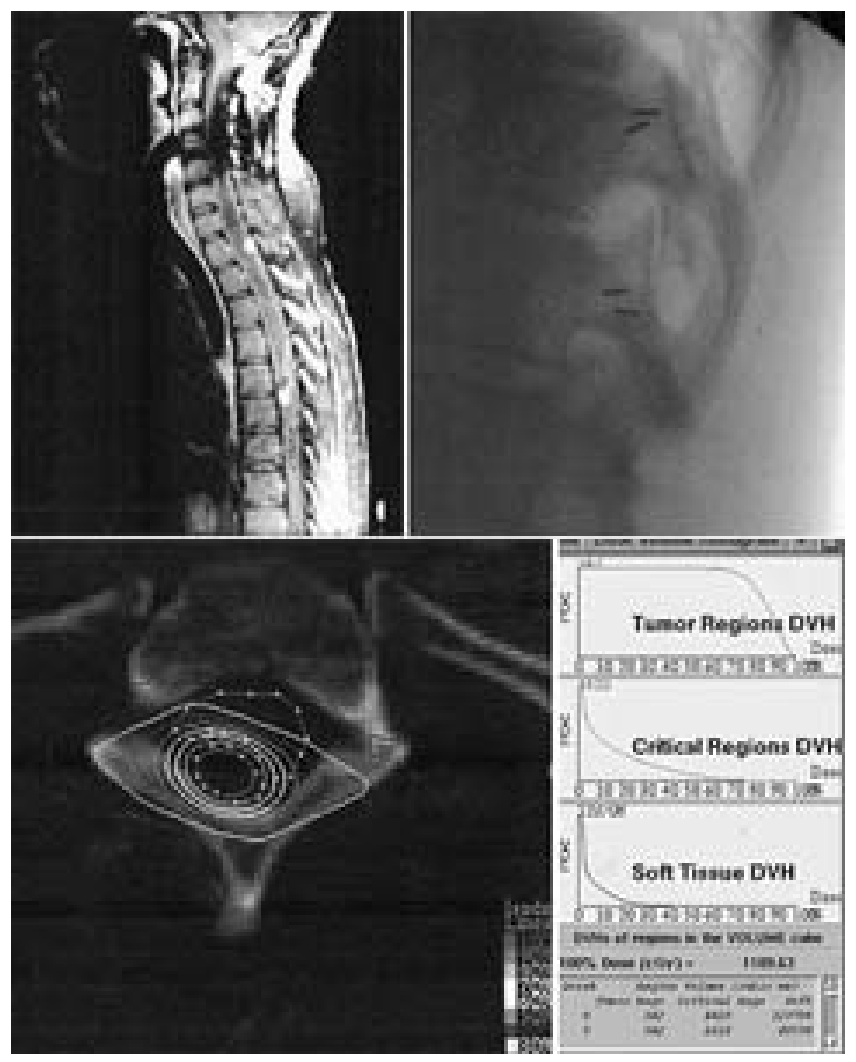

Fig. 1. Upper Left: Gadolinium-enhanced sagittal magnetic resonance image obtained in a 28 -year-old man with von Hippel-Lindau syndrome and multiple spinal hemangioblastomas. The patient refused to undergo open resection of these spinal lesions. Upper Right: Lateral radiograph of the fiducials implanted in the thoracic spine pedicles. Lower Left: Isodose lines of the treatment plan for the T-2 level tumor. The $80 \%$ isodose represents the prescribed dose of $1250 \mathrm{cGy}$, the tumor volume is $0.34 \mathrm{ml}$, and $0.02 \mathrm{ml}$ of the spinal cord received greater than $800 \mathrm{cGy}$. Notice the conformality around the tumor. Lower Right: Dose volume histogram demonstrating that $83.3 \%$ of the tumor volume received $80 \%$ of the maximum dose of $1250 \mathrm{cGy}$.

had received prior external-beam irradiation with maximum spinal cord doses. Tumor volume ranged from 0.3 to $168 \mathrm{ml}$ (mean $26.7 \mathrm{ml}$ ). Tumors were outlined based on CT scanning. An inverse treatment planning technique was used such that the tumor received the maximum dose allowable with the restriction of the maximum spinal cord tolerance dose, as well as other critical structures such as small bowel and kidneys.

\section{Clinical Evaluation}

All patients successfully completed the treatment in an outpatient setting. Thirty-one patients were treated for significant lesion-related pain. At 1-month follow-up examination, axial and radicular pain had improved in all patients who were symptomatic prior to treatment.

A single durotomy occurred during the percutaneous fiducial placement procedure. This patient required no further intervention, and she never experienced low-pressure headaches. Patients were evaluated again at 30 days for treatment-related complications. One patient, who had harbored an upper cervical lesion, experienced a transient episode of parotitis, a rare but well-described complication of irradiation. Another patient with a cervical lesion experienced a linear occipital hair loss thought to be due to one of the radiation paths. Mild, transient nausea was demonstrated in several patients with lumbar lesions, which was thought due to radiation affecting the stomach and small bowel. Later in the series, the dose to the bowel was limited to avoid this complication. No radiation-induced myelopathy was detected in this series.

\section{DISCUSSION}

Standard treatments for spinal tumors include radiotherapy alone; radionuclide therapy; radiotherapy combined with systemic chemotherapy; hormonal therapy; or surgical decompression and/or stabilization followed by radiotherapy. ${ }^{15}$ When a spinal tumor causes compression of the spinal cord or other neural elements, decompression is often necessary-with or without spinal fixation based on the extent of spinal column destruction and instability of the spine. The goals of local radiotherapy in the treatment of spinal tumors have been palliation, prevention of pathological fractures, and halting progression of or reversing neurological compromise.

Conventional external-beam radiotherapy for spinal tumors is usually administered through a course of 10 to 20 daily fractions to a total dose of 3000 to $4000 \mathrm{cGy}$. In conventional radiotherapy a primary factor limiting the radiation dose in this tumor control is the low tolerance of the spinal cord to radiation. Authors of recent studies involving hypofractionated or single-dose treatments for spinal metastases have reported results that were comparable with those achieved using conventional fractionation. ${ }^{10,19}$ Furthermore, hypofractionation or single-dose radiation decreases the treatment duration, is more convenient for patients, and is less costly.

The use of multiple radiation beams requires extremely precise control of patient position and movement of the LINAC. Current stereotactic radiotherapy surgery is limited to cases of intracranial disease because precise localization can be achieved only by neurosurgical frames fixed to the patient's skull. Most radiosurgery procedures involve the fixation of a head frame device that is attached by four screws inserted into the patient's skull. This device ensures precise targeting of the radiation to the tumor. As a corollary, treatment is typically limited to single-fraction treatments. Radiosurgery has been reported in the treatment of many different intracranial lesions, both benign and malignant, and is widely accepted as safe and effective. $5,9,20,27,28,34$

Stereotactic radiosurgery offers a method for delivering high-dose radiation in a single or limited number of fractions to a small volume encompassing the tumor while minimizing the dose to adjacent normal structures. The authors of a study originating from the University of Arizona in 1996 demonstrated that spinal stereotactic radiosurgery involving the use of a body frame was both feasible and safe. ${ }^{17}$ Precise targeting of the tumor, however, required that the spine be fixed using a large frame in which clamps were applied to the spinous processes. Multiple 1- to 2-cm incisions were necessary, after induc- 
tion of general anesthesia. Although the authors concluded that radiosurgery was beneficial, the overall treatment strategy was associated with risks of spinous process fracture, infection, delayed wound healing, and anesthesiarelated complication.

In one recent article the authors reviewed their experience using the CyberKnife for spinal lesions at Stanford University. ${ }^{33}$ Sixteen patients with a variety of spinal lesions including vascular malformations underwent both stereotactic radiosurgery and hypofractionated stereotactic radiotherapy. The lesions were all believed to be surgically inaccessible. There were no complications related to the procedure or progression of disease in a minimum 6-month follow-up period. The technique used at our institution is adapted from the Stanford experience.

There is no large-scale study to date involving spinal radiosurgery in which optimal radiation doses have been developed for this treatment technique. There is also no published experience regarding the tolerance of the human spinal cord to such single-fraction doses. In a review evaluating 172 patients who underwent cervical and thoracic fractionated radiotherapy at the University of California at San Francisco (total dose 4000-7000 cGy fractionated extending 2-3 weeks), Wara, et al., ${ }^{35}$ reported nine cases of radiation-induced myelopathy. Three of nine patients experienced mild cervical cord-related neurological deficits without significant long-term symptoms. The length of the spinal cord exposed to radiation ranged from 4 to $22 \mathrm{~cm}$. Hatlevoll, et al., ${ }^{18}$ reported a series of 387 patients with bronchial carcinoma treated with a split-course regimen involving large single-fractions. Seventeen patients developed radiation myelitis, with a mean total dose of 3800 cGy. Kim and Fayos ${ }^{21}$ reported seven patients with transverse myelopathy of 109 patients treated with definitive radiotherapy for head and neck cancer; the total dose ranged from 5700 to $6200 \mathrm{cGy}$, and the mean field size of $10 \times 10 \mathrm{~cm}$. Abbatucci, et al., ${ }^{1}$ reported eight cases of radiation-induced myelopathy in a series of 203 patients in whom the total radiation dose ranged from 5400 to $6000 \mathrm{cGy}$ targeting the cervical and thoracic spine. McCunniff and Liang ${ }^{29}$ reported only one case of radiation-induced myelopathy of 652 patients who had received greater than 6000 cGy. Phillips and Buschke ${ }^{32}$ reported three cases of transverse myelitis in 350 patients with chest tumors in whom the total radiation dose ranged from 3300 to $4350 \mathrm{cGy}$. Based on the aforementioned series, the incidence of radiation-induced myelopathy in series involving conventionally fractionated radiotherapy in patients with cervical and thoracic disease ranged from 0.2 to $5 \%$.

Unlike conventional radiotherapy in which a full dose is delivered to both the vertebral body and the spinal cord, the CyberKnife can deliver a single high-dose radiation fraction to the target tissue while sparing most of the adjacent spinal cord. The treatment plan can create a high gradient dose falloff to the target tissue, which should significantly reduce the possibility of radiation-induced myelopathy. This is the main advantage of stereotactic radiosurgery for treatment of metastatic spinal tumors.

Tumors of the spine affect a large number of cancer patients each year and result in significant pain, destruction of the spinal column causing significant mechanical instability, and neurological deficits. Standard therapeutic op- tions include surgery and fractionated external-beam radiotherapy. The first option can be associated with significant morbidity and limited local tumor control. Radiotherapy may provide less than optimal pain relief because the total dose is limited by the tolerance of adjacent tissues (for example, spinal cord). Therefore, improved treatment would provide the patient with a better quality of life. The proposed treatment represents a logical extension of the current state-of-the-art radiotherapy. It has the potential to improve significantly local control of cancer of the spine, which could translate into more effective palliation and potentially longer survival. Another advantage to the patient is that irradiation can be completed in a single day rather than extending several weeks, which is not inconsequential for patients with a limited life expectancy. In addition, cancer patients may have difficulty with access to a radiation treatment facility for prolonged, daily fractionated therapy. Finally, the procedure is noninvasive minimally invasive and can be performed in an outpatient setting.

Stereotactic radiosurgery has traditionally been overseen by "stereotactic and functional" neurosurgeons. In the past, stereotactic radiosurgery and spinal surgery were mutually exclusive. In the recent past, spinal oncology has focused on the application of new instrumentation to spinal reconstruction, improved surgical approaches, new radiation delivery systems (for example, brachytherapy), and surgery-related complication avoidance. In this study we found that stereotactic radiosurgery now has a feasible and safe delivery system available for the treatment of spinal lesions.

\section{CONCLUSIONS}

Spinal stereotactic radiosurgery was found to be both feasible and safe. The major potential benefits of radiosurgical ablation of spinal lesions are relatively short treatment time in an outpatient setting combined with potentially better local control of the tumor with minimal risk of side effects. Such an outcome could translate into better palliation of symptoms and longer survival period. In addition, this procedure will allow for the treatment of lesions previously irradiated using external-beam radiotherapy. This new procedure offers an alternative therapeutic modality for the treatment of spinal neoplasms in medically inoperable patients, previously irradiated sites, and for lesions not amenable to open surgical techniques, or as an adjunct to surgery. Current efforts are underway to collect outcome data in this patient population to allow for a more accurate assessment of the clinical efficacy of this new treatment modality.

\section{References}

1. Abbatucci JS, Delozier T, Quint R, et al: Radiation myelopathy of the cervical spinal cord: time, dose and volume factors. Int J Radiat Oncol Biol Phys 4:239-248, 1978

2. Adler JR, Cox RS, Kaplan I, et al: Stereotactic radiosurgical treatment of brain metastases. J Neurosurg 76:444-449, 1992

3. Adler JR Jr, Chang SD, Murphy MJ, et al: The Cyberknife: a frameless robotic system for radiosurgery. Stereotact Funct Neurosurg 69:124-128, 1997

4. Adler JR Jr, Murphy MJ, Chang SD, et al: Image-guided robotic radiosurgery. Neurosurgery 44:1299-1307, 1999 
5. Alexander E III, Loeffler JS, Lunsford LD: Stereotactic Radiosurgery. New York: McGraw-Hill, 1993

6. Backlund EO, Ganz JC: Pituitary adenomas: Gamma Knife, in Alexander E III, Loeffler JS, Lunsford LD (eds): Stereotactic Radiosurgery. New York: McGraw-Hill, 1993, pp 167-173

7. Chang SD, Adler JR Jr: Current status and optimal use of radiosurgery. Oncology 15:209-221, 2001

8. Chang SD, Adler JR Jr, Hancock SL: The clinical uses of radiosurgery. Oncology 12:1181-1191, 1998

9. Chang SD, Murphy MJ, Martin DP, et al: Image-guided robotic radiosurgery: clinical and radiographic results with the CyberKnife, in Kondziolka D (ed): Radiosurgery. Basel: Karger, 2000, pp 22-33

10. Colombo F, Benedetti A, Pozza F, et al: Stereotactic radiosurgery utilizing a linear accelerator. Appl Neurophysiol 48: 133-145, 1985

11. Colombo F, Pozza F, Chierego G: Linear accelerator radiosurgery of cerebral arteriovenous malformations: an update. Neurosurgery 34:14-21, 1994

12. Faul CM, Flickinger JC: The use of radiation in the management of spinal metastases. J Neurooncol 23:149-161, 1995

13. Flickinger JC, Kondziolka D, Lunsford LD, et al: A multi-institutional experience with stereotactic radiosurgery for soliatary brain metastasis. Int J Rad Onc Biol Phys 28:797-802, 1994

14. Friedman WA, Bova FJ, Mendenhall WM: Linear accelerator radiosurgery for arteriovenous malformations: the relationship of size to outcome. J Neurosurg 82:180-189, 1995

15. Gerszten PC, Welch WC: Current surgical management of metastatic spinal disease. Oncology 14:1013-1024, 2000

16. Guthrie BL, Adler JR Jr: Computer-assisted preoperative planning, interactive surgery, and frameless stereotaxy. Clin Neurosurg 38:112-131, 1991

17. Hamilton AJ, Lulu BA, Fosmire H, et al: Preliminary clinical experience with linear accelerator-based spinal stereotactic radiosurgery. Neurosurgery 36:311-319, 1995

18. Hatlevoll R, Host H, Kaalhus O: Myelopathy following radiotherapy of bronchial carcinoma with large single fractions: a retrospective study. Int J Radiat Oncol Biol Phys 9:41-44, 1983

19. Hitchcock E, Kitchen G, Dalton E, et al: Stereotactic LINAC radiosurgery. Br J Neurosurg 3:305-312, 1989

20. Kaplan ID, Adler JR, Hicks WL Jr, et al: Radiosurgery for palliation of base of skull recurrences from head and neck cancers. Cancer 70:1980-1984, 1992

21. Kim YH, Fayos JV: Radiation tolerance of the cervical spinal cord. Radiology 139:473-478, 1981

22. Kondziolka D, Lunsford LD, McLaughlin MR, et al: Long-term outcomes after radiosurgery for acoustic neuromas. N Engl J Med 339:1426-1433, 1998

23. Kondziolka D, Niranjan A, Lunsford LD, et al: Stereotactic radiosurgery for meningiomas. Neurosurg Clin N Am 10: 317-325, 1999

24. Kondziolka D, Patel A, Lunsford LD, et al: Stereotactic radiosurgery plus whole brain radiotherapy versus radiotherapy alone for patients with multiple brain metastases. Int J Radiat Oncol Biol Phys 45:427-434, 1999

25. Loblaw DA, Laperriere NJ: Emergency treatment of malignant extradural spinal cord compression: an evidence-based guideline. J Clin Oncol 16:1613-1624, 1998

26. Loeffler JS, Alexander E III: Radiosurgery for the treatment of intracranial metastasis, in Alexander E III, Loeffler JS, Lunsford D (eds): Radiosurgery. New York: McGraw-Hill, 1993, pp 197-206

27. Loeffler JS, Kooy HM, Wen PY, et al: The treatment of recurrent brain metastases with stereotactic radiosurgery. J Clin Oncol 8:576-582, 1990

28. Lunsford LD, Flickinger J, Coffey, RJ: Stereotactic gamma knife radiosurgery. Initial North American experience in 207 patients. Arch Neurol 47:169-175, 1990

29. McCunniff AJ, Liang MJ: Radiation tolerance of the cervical spinal cord. Int J Radiat Oncol Biol Phys 16:675-678, 1989

30. Murphy MJ, Chang S, Gibbs I, et al: Image-guided radiosurgery in the treatment of spinal metastases. Neurosurg Focus 11(6): Article 6, 2001

31. Murphy MJ, Cox RS: The accuracy of dose localization for an image-guided frameless radiosurgery system. Med Phys 23: 2043-2049, 1996

32. Phillips TL, Buschke F: Radiation tolerance of the thoracic spinal cord. Am J Roentgenol Radium Ther Nucl Med 105: 659-664, 1969

33. Ryu SI, Chang SD, Kim DH, et al: Image-guided hypo-fractionated stereotactic radiosurgery to spinal lesions. Neurosurgery 49:838-846, 2001

34. Steiner L, Lindquist C, Adler JR, et al: Clinical outcome of radiosurgery for cerebral arteriovenous malformations. J Neurosurg 77:1-8, 1992

35. Wara WM, Phillips TL, Sheline GE, et al: Radiation tolerance of the spinal cord. Cancer 35:1558-1562, 1975

Manuscript received August 15, 2002.

Accepted in final form September 13, 2002.

This study was supported by a research grant awarded by the American Association of Neurological Surgeons and Congress of Neurological Surgeons Section on Disorders of the Spine and Peripheral Nerves.

Address reprint requests to: Peter C. Gerszten, M.D., M.P.H., Department of Neurological Surgery, Presbyterian University Hospital, Suite B-400, 200 Lothrop Street, Pittsburgh, Pennsylvania 15213. email: gerszten@neuronet.pitt.edu. 